\title{
It is time to bring retinopathy of prematurity monitoring into the 21st century
}

\author{
Michael T. Trese
}

Received: 31 October 2014 / Accepted: 3 November 2014 / Published online: 4 December 2014

(C) Springer-Verlag Berlin Heidelberg 2014

The subjective nature of clinical ophthalmology is perhaps nowhere more demonstrable than in the determination of plus disease and aggressive posterior retinopathy of prematurity (APROP) [1-3], in part because the clinical determination of plus disease and the number of vessels in zone 1 is often difficult to determine in a squirming infant. Even photographic screening to detect plus disease and aggressive posterior retinopathy of prematurity has its subjective features, as noted in the article entitled, "Aggressive posterior retinopathy of prematurity: Quantitative analysis of vascular features," in this issue of Graefes' Archives for Clinical and Experimental Ophthalmology. The determination of plus disease and number of clock-hours of vessels in zone 1 can predict a rapid course to severe retinopathy of prematurity (ROP), with retinal detachment and possible lifelong blindness. The higher the number of clock-hours of vessels in zone 1, the greater the likelihood of progressive disease. A standardized computerized method of detection of plus disease and exact sizing of zone 1 disease based on photographic imaging would be helpful in allowing the clinician to study the images carefully and provide more accurate management for the infant.

In the study in this issue of von Graefes' Archives, a single posterior pole picture was presented to readers. Does this really represent enough data, or would a montaged image of more of the retina with complete zone 1 determination at least give the readers more confidence of their opinion? It seems that more objective plus disease determination and rapidity of treatment would have a positive effect on the outcomes. The ability to use images from wide-angle cameras and computerized software to reach an objective determination of APROP and plus disease may move ROP treatment forward in a global fashion $[4,5]$. As ROP experts are in short supply worldwide, and the medical malpractice risk of ROP drives doctors further and further from ROP care in much of the world, such a determination with computerized aids may afford less experienced doctors a higher level of confidence to be able to supply accurate interpretation of ROP images.

Although further validation studies are needed, the authors are to be congratulated for the excellent pilot study, which shows us the way for the future for ROP care. This type of photographic documentation and computer-linked vascular measurement can create a global standard of care that will revolutionize ROP management and reduce blindness for children that are at risk worldwide.

\section{References}

1. Wallace DK, Quinn GE, Freedman SF et al (2008) Agreement among pediatric ophthalmologists in diagnosing plus and pre-plus disease in retinopathy of prematurity. J AAPOS 12:352-356

2. Slidsborg C, Forman JL, Fielder AR et al (2012) Experts do not agree when to treat retinopathy of prematurity based on plus disease. $\mathrm{Br} \mathrm{J}$ Ophthalmol 96:549-553

3. Chiang MF, Gelman R, Williams SL et al (2008) Plus disease in retinopathy of prematurity: development of composite images by quantification of expert opinion. Invest Ophthalmol Vis Sci 49: 4064-4070

4. Swanson C, Cocker KD, Parker KH et al (2003) Computerized analysis of retinal vessel width and tortuosity in premature infants. Invest Ophthalmol Vis Sci 49:3577-3585

5. Wallace DK, Zhao Z, Freedman SF (2007) A pilot study using "ROPtool" to quantify plus disease in retinopathy of prematurity. J AAPOS 11:381-387
M. T. Trese $(\square)$

3535 W. 13 Mile Road, Suite 344, Royal Oak, MI 48073, USA

e-mail: mgjt46@aol.com 